ANNALES

POLONICI MATHEMATICI

$88.1(2006)$

\title{
Invariance in the class of weighted quasi-arithmetic means
}

\author{
by JUSTYNA JARCZYK (Zielona Góra) and \\ JANUSZ MatKowski (Zielona Góra and Katowice)
}

\begin{abstract}
Under the assumption of twice continuous differentiability of some of the functions involved we determine all the weighted quasi-arithmetic means $M, N, K$ such that $K$ is $(M, N)$-invariant, that is, $K \circ(M, N)=K$. Some applications to iteration theory and functional equations are presented.
\end{abstract}

1. Introduction. In the whole paper $I \subset \mathbb{R}$ denotes an interval. A function $M: I^{2} \rightarrow \mathbb{R}$ is said to be a mean on $I$ if

$$
\min (x, y) \leq M(x, y) \leq \max (x, y), \quad x, y \in I .
$$

If $M: I^{2} \rightarrow \mathbb{R}$ is a mean, then $M$ is reflexive, that is, $M(x, x)=x$ for all $x \in I$ and, consequently, for every interval $J \subset I$ we have $M\left(J^{2}\right)=J$; in particular, $M\left(I^{2}\right)=I$.

If $\alpha: I \rightarrow \mathbb{R}$ is a continuous strictly monotonic function and $p \in(0,1)$ then $A_{p}^{[\alpha]}: I^{2} \rightarrow I$, given by

$$
A_{p}^{[\alpha]}(x, y):=\alpha^{-1}(p \alpha(x)+(1-p) \alpha(y)), \quad x, y \in I,
$$

is a mean; it is called a weighted quasi-arithmetic mean with generator $\alpha$ and weight $(p, 1-p)$. In the case $\alpha=\left.\mathrm{id}\right|_{I}$ the mean $A_{p}^{[\alpha]}$ becomes the weighted arithmetic mean and is denoted by $A_{p}$; thus $A_{p}(x, y)=p x+(1-p) y$. We write simply $A$ instead of $A_{1 / 2}$.

Let $M, N: I^{2} \rightarrow I$ be means. A mean $K: I^{2} \rightarrow I$ is said to be invariant with respect to $(M, N)$, briefly $(M, N)$-invariant, or $K$ is called the Gauss composition of $M$ and $N$ (cf. [3], [5]), if $K \circ(M, N)=K$.

Fix $p, q, r \in(0,1)$. Assuming twice continuous differentiability of some of the functions involved we determine all triples $(\alpha, \beta, \gamma)$ and $(p, q, r)$ for

2000 Mathematics Subject Classification: Primary 26E60; Secondary 39B22. mean.

Key words and phrases: mean, functional equation, invariant mean, quasi-arithmetic 
which the weighted quasi-arithmetic mean $A_{p}^{[\alpha]}$ is $\left(A_{q}^{[\beta]}, A_{r}^{[\gamma]}\right)$-invariant, i.e.

$$
A_{p}^{[\alpha]} \circ\left(A_{q}^{[\beta]}, A_{r}^{[\gamma]}\right)=A_{p}^{[\alpha]}
$$

(see Theorem 2 in Section 4).

The case when $p=q=r=1 / 2$ (called simply the case of quasiarithmetic means) has a long history. The analytic solutions were found by O. Sutô in 1914 [7]. The twice continuously differentiable solutions are given in [5]. Moreover, continuously differentiable solutions were found by Z. Daróczy and Zs. Páles [2], and finally, without any regularity assumption, the problem was solved by Z. Daróczy and Zs. Páles [3] (cf. also [1] as well as [3] for further references).

The fundamental role for Theorem 2 will be played here by Theorem 1 in Section 4, concerning the case of $\alpha$ being the identity function. In the proof of Theorem 1 we need a characterization of conditionally homogeneous weighted quasi-arithmetic means given by Proposition 1 proved in Section 2 and by the lemmas of Section 3 .

In Section 5 we also apply Theorem 1 to establish the limit of the iteration sequence of some mean type mappings (Remark) and to solve a functional equation (Theorem 3).

2. Conditional homogeneity of the quasi-arithmetic mean. Denote by $C M(I)$ the class of all continuous strictly monotonic functions defined on $I$. In the proof of Theorem 1 we need the following

Proposition 1. Assume that $I \subset(0, \infty)$. Let $q \in(0,1)$ and $\sigma \in C M(I)$. The mean $A_{q}^{[\sigma]}$ is conditionally homogeneous, i.e.

$$
A_{q}^{[\sigma]}(s x, s y)=s A_{q}^{[\sigma]}(x, y)
$$

for all $x, y \in I$ and $s>0$ with sx, sy $\in I$ if, and only if, there are $a \in \mathbb{R} \backslash\{0\}$ and $b \in \mathbb{R}$ such that either

$$
\sigma(x)=a x^{\eta}+b, \quad x \in I,
$$

for some $\eta \in \mathbb{R} \backslash\{0\}$, or

$$
\sigma(x)=a \ln x+b, \quad x \in I .
$$

Before proving this proposition we will find the form of so-called conditional local groups of continuous affine maps.

Proposition 2. Let $\left\{X_{s}\right\}_{s \in S}$, where $S \subset \mathbb{R}$ is an interval containing 0 , be a family of subsets of $\mathbb{R}$ and let $F: \bigcup_{s \in S}\{s\} \times X_{s} \rightarrow \mathbb{R}$. Assume that $F(\cdot, u)$ and $F(\cdot, v)$ are continuous for some different $u, v \in \bigcap_{s \in S} X_{s}$ and $F(s, \cdot)$ is continuous affine for every $s \in S$. Assume also that for every $s, t \in S$ with $s+t \in S$ there is an at least two-element set $U_{s, t} \subset X_{s} \cap X_{s+t}$ 
such that

$$
F\left(\{s\} \times U_{s, t}\right) \subset X_{t}
$$

and

$$
F(s+t, u)=F(t, F(s, u)), \quad u \in U_{s, t} .
$$

Then either $F$ is constant, or there is an interval $S_{0} \subset S$ containing 0 and such that

$$
F(s, u)=c s+u, \quad s \in S_{0}, u \in X_{s}
$$

with a $c \in \mathbb{R}$, or

$$
F(s, u)=c_{1}^{s}\left(u-c_{2}\right)+c_{2}, \quad s \in S_{0}, u \in X_{s},
$$

with some $c_{1} \in(0, \infty)$ and $c_{2} \in \mathbb{R}$.

Proof. Let $m: S \rightarrow \mathbb{R}$ and $k: S \rightarrow \mathbb{R}$ be such that

$$
F(s, u)=m(s) u+k(s), \quad s \in S, u \in X_{s} .
$$

Taking any $u, v \in \bigcap_{s \in S} X_{s}, u \neq v$, such that $F(\cdot, u)$ and $F(\cdot, v)$ are continuous we see that $m$ and $k$ are continuous as linear combinations of $F(\cdot, u)$ and $F(\cdot, v)$.

Fix any $s, t \in S$ with $s+t \in S$ and choose a $U_{s, t} \subset X_{s} \cap X_{s+t}$ with at least two points, satisfying (2) and (3). Taking an arbitrary $u \in U_{s, t}$ and using (6), (3), and again (6), we get

$$
\begin{aligned}
m(s+t) u+k(s+t) & =F(s+t, u)=F(t, F(s, u)) \\
& =m(t)(m(s) u+k(s))+k(t) \\
& =m(s) m(t) u+m(t) m(s)+k .
\end{aligned}
$$

Consequently,

$$
m(s+t)=m(s) m(t)
$$

and

$$
k(s+t)=m(t) k(s)+k(t)
$$

for all $s, t \in S$ with $s+t \in S$.

Assume that $m\left(s_{0}\right)=0$ for some $s_{0} \in S$ with $-s_{0} \in S$. By (7) we get

$$
m(0)=m\left(s_{0}\right) m\left(-s_{0}\right)=0,
$$

whence, again according to $(7)$, we have $m(s)=m(s) m(0)=0$ for all $s \in S$ and, consequently, $k$ is constant by (8). Then, on account of (6) also $F$ is constant. Now we can assume that $m$ does not vanish in a subinterval $S_{0} \subset S$ containing 0 and, in addition, satisfying $S_{0}+S_{0} \subset S$. Then, by (7), $\left.m\right|_{S_{0}}$ is positive.

Since (7) is a multiplicative version of the restricted Cauchy equation, with the use of continuity arguments and making use of [4, Theorem 2, 
p. 327] we infer that there exists a $c_{1} \in(0, \infty)$ such that $m(s)=c_{1}^{s}$ for all $s \in S_{0}$.

If $c_{1}=1$ it follows from (8) that $k(s+t)=k(s)+k(t)$ for all $s, t \in S_{0}$ and, again by [4, Theorem 2, p. 327], we find a $c \in \mathbb{R}$ such that $k(s)=c s$ for all $s \in S_{0}$. Consequently, on account of (6) we have $F(s, u)=c s+u$ for all $s \in S_{0}$ and $u \in X_{s}$.

Now assume that $c_{1} \neq 1$. Then, by $(8), k(s+t)=c_{1}^{t} k(s)+k(t)$ for all $s, t \in S_{0}$. By symmetry $k(s+t)=c_{1}^{s} k(t)+k(s)$ and

$$
\frac{k(s)}{1-c_{1}^{s}}=\frac{k(t)}{1-c_{1}^{t}}, \quad s, t \in S_{0} \backslash\{0\} .
$$

Thus there exists a $c_{2} \in \mathbb{R}$ such that $k(s)=c_{2}\left(1-c_{1}^{s}\right)$ for all $s \in S_{0}$. Consequently, by (6), we have $F(s, u)=c_{1}^{s}\left(u-c_{2}\right)+c_{2}$ for all $s \in S_{0}$ and $u \in X_{s}$.

Proof of Proposition 1. Without loss of generality we may assume that int $I \neq \emptyset$.

Assume that $A_{q}^{[\sigma]}$ is conditionally homogeneous, that is,

$$
\sigma^{-1}(q \sigma(s x)+(1-q) \sigma(s y))=s \sigma^{-1}(q \sigma(x)+(1-q) \sigma(y))
$$

for all $x, y \in I$ and $s>0$ with $s x, s y \in I$. Fix an $x_{0} \in \operatorname{int} I$ and put $u_{0}=\sigma\left(x_{0}\right)$. Then $u_{0} \in \operatorname{int} \sigma(I)$ and there exist $\delta>0$ and $\delta_{0}>1$ such that

$$
\sigma\left(s x_{0}\right) \in\left(u_{0}-\delta, u_{0}+\delta\right)
$$

for all $s \in\left(1 / \delta_{0}, \delta_{0}\right)$ and $s \sigma^{-1}(u) \in I$ for all $s \in\left(1 / \delta_{0}, \delta_{0}\right)$ and $u \in\left(u_{0}-\delta\right.$, $\left.u_{0}+\delta\right)$. In particular, $\left(u_{0}-\delta, u_{0}+\delta\right) \subset \sigma(I)$. Put $S=\left(-\ln \delta_{0}, \ln \delta_{0}\right)$. Defining $F: S \times\left(u_{0}-\delta, u_{0}+\delta\right) \rightarrow \mathbb{R}$ by

$$
F(s, u)=\sigma\left(e^{s} \sigma^{-1}(u)\right)
$$

we can rewrite (9) in the form

$$
\begin{aligned}
F(s, q u+(1-q) v)=q F(s, u) & +(1-q) F(s, v), \\
s & \in S, u, v \in\left(u_{0}-\delta, u_{0}+\delta\right) .
\end{aligned}
$$

Fix an $s \in S$. Then, applying the Daróczy-Páles identity

$$
q\left((1-q) \frac{u+v}{2}+q u\right)+(1-q)\left(q \frac{u+v}{2}+(1-q) v\right)=q u+(1-q) v,
$$

we get

$$
\begin{aligned}
F\left(s, q\left((1-q) \frac{u+v}{2}+q u\right)+(1-q)(q\right. & \left.\left.\left.\frac{u+v}{2}+(1-q) v\right)\right)\right) \\
& =q F(s, u)+(1-q) F(s, v)
\end{aligned}
$$


for all $u, v \in\left(u_{0}-\delta, u_{0}+\delta\right)$. Now, applying (11) twice to the left-hand side expression, for all $u, v \in\left(u_{0}-\delta, u_{0}+\delta\right)$ we have

$q^{2} F(s, u)+2 q(1-q) F\left(s, \frac{u+v}{2}\right)+(1-q)^{2} F(s, v)=q F(s, u)+(1-q) F(s, v)$ and, consequently,

$$
2 F\left(s, \frac{u+v}{2}\right)=F(s, u)+F(s, v), \quad u, v \in\left(u_{0}-\delta, u_{0}+\delta\right) .
$$

By the continuity of $F$ and the Jensen theorem we can find $m(s), k(s) \in \mathbb{R}$ such that

$$
F(s, u)=m(s) u+k(s), \quad u \in\left(u_{0}-\delta, u_{0}+\delta\right) .
$$

Thus we have shown that $F(s, \cdot)$ is continuous affine for every $s \in S$. Moreover, if $s, t, s+t \in S$ then, by (10), we have $F\left(s, u_{0}\right) \in\left(u_{0}-\delta, u_{0}+\delta\right)$, whence

$$
F(s, u) \in\left(u_{0}-\delta, u_{0}+\delta\right)
$$

for $u$ from a neighbourhood $U_{s, t} \subset\left(u_{0}-\delta, u_{0}+\delta\right)$ of $u_{0}$ and

$$
\left.F(t, F(s, u))=\sigma\left(e^{t} \sigma^{-1} \sigma\left(e^{s} \sigma^{-1}(u)\right)\right)\right)=\sigma\left(e^{s+t} \sigma^{-1}(u)\right)=F(s+t, u)
$$

for all $u \in U_{s, t}$. Since $F$ is not constant, on account of Proposition 2 there is an interval $S_{0} \subset S$ containing 0 and such that $F$ is of the form (4) or (5), where $c_{1} \in(0, \infty)$ and $c_{2}, c \in \mathbb{R}$.

In this way we have shown that every point $x$ of int $I$ has a neighbourhood in which $\sigma$ has one of the following forms:

$$
\sigma(x)=a \ln x+b, \quad \sigma(x)=a x^{\eta}+b
$$

with $a \in \mathbb{R} \backslash\{0\}, b \in \mathbb{R}$ and $\eta \in \mathbb{R} \backslash\{0\}$ depending on that point. By standard arguments we deduce that one of these forms holds on the whole interval $I$.

The converse assertion is obvious.

3. Auxiliary results. Denote by $C^{n} M(I)$ the subclass of $C M(I)$ consisting of functions which are $n$-times continuously differentiable.

LEMMA 1. Let $p, q, r \in(0,1)$. If $\varphi, \psi \in C^{1} M(I)$ satisfy

$$
p A_{q}^{[\varphi]}(x, y)+(1-p) A_{r}^{[\psi]}(x, y)=p x+(1-p) y
$$

and $\varphi^{\prime}(x) \neq 0, \psi^{\prime}(x) \neq 0$ for all $x \in I$, then

$$
p=\frac{r}{1-q+r} \text {. }
$$

Proof. Differentiation of both sides of (12) with respect to $x$ gives

$$
p \frac{q \varphi^{\prime}(x)}{\varphi^{\prime}\left(A_{q}^{[\varphi]}(x, y)\right)}+(1-p) \frac{r \psi^{\prime}(x)}{\psi^{\prime}\left(A_{r}^{[\psi]}(x, y)\right)}=p, \quad x, y \in I
$$


Letting $y \rightarrow x$ and taking into account the reflexivity of the means we obtain the desired equality.

Lemma 2. Let $p, q, r \in(0,1)$. If $\varphi, \psi \in C^{1} M(I)$ satisfy $(12)$ and $\varphi^{\prime}(x) \neq 0$ and $\psi^{\prime}(x) \neq 0$ for all $x \in I$, then $f:=\varphi^{\prime} \circ \varphi^{-1}$ and $g:=\psi^{\prime} \circ \varphi^{-1}$ satisfy the equation

$$
\begin{aligned}
& f(q u+(1-q) v)[(1-r) g(v)-(1-q) g(u)] \\
& \quad=q(1-r) f(u) g(v)-r(1-q) f(v) g(u)
\end{aligned}
$$

for all $u, v \in \varphi(I)$.

Proof. Differentiating both sides of (12), first with respect to $x$ and then with respect to $y$, we get (13) and

$$
p \frac{(1-q) \varphi^{\prime}(y)}{\varphi^{\prime}\left(A_{q}^{[\varphi]}(x, y)\right)}+(1-p) \frac{(1-r) \psi^{\prime}(y)}{\psi^{\prime}\left(A_{r}^{[\psi]}(x, y)\right)}=1-p, \quad x, y \in I .
$$

Multiplying (13) by $(1-r) \psi^{\prime}(y)$ and (15) by $r \psi^{\prime}(x)$ we get

$$
\begin{aligned}
& \frac{p q(1-r) \varphi^{\prime}(x) \psi^{\prime}(y)}{\varphi^{\prime}\left(A_{q}^{[\varphi]}(x, y)\right)}+\frac{(1-p) r(1-r) \psi^{\prime}(x) \psi^{\prime}(y)}{\psi^{\prime}\left(A_{r}^{[\psi]}(x, y)\right)}=p(1-r) \psi^{\prime}(y), \\
& \frac{p(1-q) r \varphi^{\prime}(y) \psi^{\prime}(x)}{\varphi^{\prime}\left(A_{q}^{[\varphi]}(x, y)\right)}+\frac{(1-p)(1-r) r \psi^{\prime}(x) \psi^{\prime}(y)}{\psi^{\prime}\left(A_{r}^{[\psi]}(x, y)\right)}=(1-p) r \psi^{\prime}(x),
\end{aligned}
$$

for all $x, y \in I$. Subtracting these equalities we obtain

$$
\frac{p q(1-r) \varphi^{\prime}(x) \psi^{\prime}(y)-p(1-q) r \varphi^{\prime}(y) \psi^{\prime}(x)}{\varphi^{\prime}\left(A_{q}^{[\varphi]}(x, y)\right)}=p(1-r) \psi^{\prime}(y)-(1-p) r \psi^{\prime}(x)
$$

for all $x, y \in I$. Setting here $x:=\varphi^{-1}(u)$ and $y:=\varphi^{-1}(v)$ we have

$$
\frac{p q(1-r) f(u) g(v)-p(1-q) r f(v) g(u)}{f(q u+(1-q) v)}=p(1-r) g(v)-r(1-p) g(u)
$$

for all $u, v \in \varphi(I)$. Now Lemma 1 yields the assertion.

Lemma 3. Let $q, r \in(0,1)$ and let $J \subset \mathbb{R}$ be an interval. If $f, g: J \rightarrow$ $(0, \infty)$ are continuously differentiable and satisfy (14) for all $u, v \in J$, then there exists a number $c>0$ such that

$$
f(u)^{q} g(u)^{1-r}=c, \quad u \in J .
$$

Proof. Differentiating both sides of (14) with respect to $u$ we get

$$
\begin{array}{r}
q f^{\prime}(q u+(1-q) v)[(1-r) g(v)-(1-q) g(u)]-(1-q) f(q u+(1-q) v) g^{\prime}(u) \\
=q(1-r) f^{\prime}(u) g(v)-(1-q) r f(v) g^{\prime}(u)
\end{array}
$$

for all $u, v \in J$. Letting $v \rightarrow u$ we obtain

$$
q f^{\prime}(u) g(u)=-(1-r) f(u) g^{\prime}(u), \quad u \in J .
$$


Consequently,

$$
f(u)^{q} g(u)^{1-r}=c, \quad u \in J,
$$

for some $c>0$.

Lemma 4. Let $q, r \in(0,1)$ and let $J \subset \mathbb{R}$ be an interval. If $f: J \rightarrow$ $(0, \infty)$ is continuous and satisfies the equation

$$
\begin{aligned}
f(q u+(1-q) v)\left[(1-r) f(v)^{-q /(1-r)}-(1-q) f(u)^{-q /(1-r)}\right] \\
=q(1-r) f(u) f(v)^{-q /(1-r)}-r(1-q) f(v) f(u)^{-q /(1-r)}
\end{aligned}
$$

for all $u, v \in J$, then either $f$ is constant, or $q+r=1$ and there exist $\lambda \in \mathbb{R} \backslash\{0\}$ and $b \in \mathbb{R}$ such that

$$
f(u)=\lambda(u-b), \quad u \in J .
$$

Proof. First assume that $f$ is not one-to-one. Fix $u_{0}, v_{0} \in J, u_{0}<v_{0}$, such that $f\left(u_{0}\right)=f\left(v_{0}\right)$. We will show that $f$ is constant on $\left[u_{0}, v_{0}\right]$. Suppose, on the contrary, that

$$
f(v) \neq f\left(u_{0}\right), \quad v \in\left(u_{0}, v_{0}\right) .
$$

By (16) we have

$$
(q-r) f\left(q u_{0}+(1-q) v_{0}\right)=(q-r) f\left(u_{0}\right),
$$

whence (17) gives $q=r$. Thus (16) takes the form

$$
\begin{aligned}
f(q u+(1-q) v)\left[f(u)^{q /(1-q)}-f(v)^{q /(1-q)}\right] & \\
& =q\left[f(u)^{1 /(1-q)}-f(v)^{1 /(1-q)}\right]
\end{aligned}
$$

for all $u, v \in J$. In particular, with the use of (17) and interchanging $u$ and $v$, we arrive at

$$
f\left(q u_{0}+(1-q) v\right)=f\left((1-q) u_{0}+q v\right), \quad v \in\left(u_{0}, v_{0}\right) .
$$

If $q=1 / 2$ equality (18) gives

$$
f\left(\frac{u+v}{2}\right)=\frac{f(u)+f(v)}{2}, \quad u, v \in J,
$$

whence $f$ is affine, which is impossible in the case of (17) with $f\left(u_{0}\right)=f\left(v_{0}\right)$. So we may further assume that $q \neq 1 / 2$. Let, for instance, $q \in(0,1 / 2)$. Fix a $u \in\left(u_{0}, q u_{0}+(1-q) v_{0}\right)$. Put $u_{1}:=u$ and

$$
u_{n+1}:=(1-q) u_{0}+q \frac{u_{n}-q u_{0}}{1-q}, \quad n \in \mathbb{N} .
$$

Clearly,

$$
u_{n}=q u_{0}+(1-q) \frac{u_{n}-q u_{0}}{1-q}, \quad n \in \mathbb{N} .
$$


Using induction we deduce from (20) that $u_{n}>u_{0}$ for all $n \in \mathbb{N}$. This implies that

$$
\frac{u_{n}-q u_{0}}{1-q}>u_{0}, \quad n \in \mathbb{N} .
$$

Hence, from (20), (21) and the condition $q<1 / 2$ we infer that the sequence $\left(u_{n}\right)_{n \in \mathbb{N}}$ is decreasing and, in particular,

$$
\frac{u_{n}-q u_{0}}{1-q} \in\left(u_{0}, v_{0}\right), \quad n \in \mathbb{N} .
$$

Consequently, by $(20)$ the sequence $\left(u_{n}\right)_{n \in \mathbb{N}}$ converges to $u_{0}$ and, according to $(19)-(21)$,

$$
f\left(u_{0}\right)=\lim _{n \rightarrow \infty} f\left(u_{n}\right)=f\left(u_{1}\right)=f(u),
$$

which, by (17), is impossible. This contradiction shows that there are a maximal interval $J_{0} \subset J$ with nonempty interior and a positive $c$ such that

$$
f(u)=c, \quad u \in J_{0} .
$$

We will show that $J_{0}=J$. Suppose this is not the case. For instance let $\sup J_{0}<\sup J$. Fix a $u \in J_{0}, u<\sup J_{0}$. Then it follows from (16) that we can find an $\varepsilon>0$ with

$$
\begin{aligned}
c\left[(1-r) f(v)^{-q /(1-r)}\right. & \left.-(1-q) c^{-q /(1-r)}\right] \\
& =q(1-r) c f(v)^{-q /(1-r)}-r(1-q) f(v) c^{-q /(1-r),}
\end{aligned}
$$

that is,

$$
f(v)^{q /(1-r)}=\frac{c^{1+q /(1-r)}(1-r)}{c-r f(v)}
$$

for all $v \in\left(\sup J_{0}, \sup J_{0}+\varepsilon\right)$. Putting here $d:=c^{1+q /(1-r)}(1-r)$ and $y:=f(v)$ we see that

$$
y^{q /(1-r)}=\frac{d}{c-r y}
$$

for all $y$ from an interval with nonempty interior, which is impossible. Thus $J_{0}=J$. In other words, $f$ is constant.

Now consider the case when $f$ is one-to-one. Putting $x=f(u)$ and $y=$ $f(v)$ in (16) we get, for all $x, y \in f(J)$,

$$
\begin{aligned}
f\left(q f^{-1}(x)+(1-q) f^{-1}(y)\right) & {\left[(1-r) y^{-q /(1-r)}-(1-q) x^{-q /(1-r)}\right] } \\
& =q(1-r) x y^{-q /(1-r)}-r(1-q) y x^{-q /(1-r)},
\end{aligned}
$$

which shows that the quasi-arithmetic mean $A_{q}^{\left[f^{-1}\right]}$ on $f(J)$ is conditionally homogeneous. On account of Proposition 1 there are $a \in \mathbb{R} \backslash\{0\}$ and $b \in \mathbb{R}$ such that either

$$
1^{\circ} f^{-1}(x)=a x^{\eta}+b, x \in f(J) \text {, for some } \eta \in \mathbb{R} \backslash\{0\},
$$

or

$$
2^{\circ} f^{-1}(x)=a \ln x+b, x \in f(J) .
$$


Suppose that case $2^{\circ}$ holds. Then we can find $c \in \mathbb{R} \backslash\{0\}$ and $\mu>0, \mu \neq 1$, such that

$$
f(u)=c \mu^{u}, \quad u \in J
$$

whence, by (16),

$$
\begin{aligned}
& \mu^{q u+(1-q) v}\left[(1-r) \mu^{-q v /(1-r)}-(1-q) \mu^{-q u /(1-r)}\right] \\
& \quad=q(1-r) \mu^{u} \mu^{-q v /(1-r)}-r(1-q) \mu^{v} \mu^{-q u /(1-r)}
\end{aligned}
$$

for all $u, v \in J$ and, consequently, for all $u, v \in \mathbb{R}$. Taking here $v=0$ and putting $w:=\mu^{u}$ we obtain

$(1-r) w^{q}-(1-q) w^{-q r /(1-r)}=q(1-r) w-r(1-q) w^{-q /(1-r)}, \quad w \in(0, \infty)$.

Thus, since $q>0,-q r /(1-r)<0$ and $-q /(1-r)<0$, we get $q=1$ and $-q r /(1-r)=-q /(1-r)$, which is impossible. Therefore case $1^{\circ}$ is satisfied and it follows that

$$
f(u)=c\left|u-u_{0}\right|^{\xi}, \quad u \in J
$$

with some $u_{0} \in \mathbb{R} \backslash J$ and $c \in(0, \infty), \xi \in \mathbb{R} \backslash\{0\}$. By (16) we get

$$
\begin{aligned}
\mid q u & +(1-q) v-\left.u_{0}\right|^{\xi}\left[(1-r)\left|v-u_{0}\right|^{-q \xi /(1-r)}-(1-q)\left|u-u_{0}\right|^{-q \xi /(1-r)}\right] \\
& =q(1-r)\left|u-u_{0}\right|^{\xi}\left|v-u_{0}\right|^{-q \xi /(1-r)}-r(1-q)\left|v-u_{0}\right|^{\xi}\left|u-u_{0}\right|^{-q \xi /(1-r)}
\end{aligned}
$$

for all $u, v \in J$, whence

$$
\begin{aligned}
(q u+(1-q) v)^{\xi}\left[(1-r) v^{-q \xi /(1-r)}-(1-q) u^{-q \xi /(1-r)}\right] & \\
& =q(1-r) u^{\xi} v^{-q \xi /(1-r)}-r(1-q) v^{\xi} u^{-q \xi /(1-r)}
\end{aligned}
$$

either for all $u, v \in J-u_{0}$, or for all $u, v \in u_{0}-J$, depending on whether $u_{0}<u, u \in J$, or $u_{0}>u, u \in J$. If $\xi<0$, then (23) gives

$$
\begin{aligned}
u^{-\xi} v^{-\xi}[ & \left.(1-r) v^{-q \xi /(1-r)}-(1-q) u^{-q \xi /(1-r)}\right] \\
& =(q u+(1-q) v)^{-\xi}\left(q(1-r) v^{-\left(1+\frac{q}{1-r}\right) \xi}-r(1-q) u^{-\left(1+\frac{q}{1-r}\right) \xi}\right)
\end{aligned}
$$

for all $u, v \in[0, \infty)$, whence, by setting $u=0$,

$$
q(1-r)(1-q)^{-\xi} v^{-\left(2+\frac{q}{1-r}\right) \xi}=0, \quad v \in[0, \infty),
$$

which is impossible. Therefore $\xi>0$ and now (23) gives

$$
\begin{aligned}
& (q u+(1-q) v)^{\xi}\left[(1-r) u^{q \xi /(1-r)}-(1-q) v^{q \xi /(1-r)}\right] \\
& \quad=q(1-r) u^{\left(1+\frac{q}{1-r}\right) \xi}-r(1-q) v^{\left(1+\frac{q}{1-r}\right) \xi}
\end{aligned}
$$

for all $u, v \in[0, \infty)$. Putting here $v=0$ we arrive at

$$
q^{\xi} u^{\xi}(1-r) u^{q \xi /(1-r)}=q(1-r) u^{\left(1+\frac{q}{1-r}\right) \xi}, \quad u \in[0, \infty) .
$$

Hence $q^{\xi}=q$, i.e. $\xi=1$. Thus, putting $u=0$ in (24), we get

$$
-(1-q) v(1-q) v^{q /(1-r)}=-r(1-q) v^{1+q /(1-r)}, \quad v \in[0, \infty),
$$


and, consequently, $r=1-q$. Moreover, according to (22) we have the desired affine form of $f$.

4. Main results. Now we are in a position to prove the main result of the paper. It concerns the case of $\alpha=\mathrm{id}$, when equation (1) takes the form (12).

THEOREM 1. Functions $\varphi, \psi \in C^{2} M(I)$ and numbers $p, q, r \in(0,1)$ satisfy (12) if, and only if, the following two conditions are fulfilled:

(i)

$$
p=\frac{r}{1-q+r}
$$

(ii) there exist $a, c \in \mathbb{R} \backslash\{0\}$ and $b, d \in \mathbb{R}$ such that

$$
\begin{array}{r}
\varphi(x)=a x+b, \quad \psi(x)=c x+d, \quad x \in I, \\
\text { or } p=1 / 2, q+r=1 \text { and } \\
\varphi(x)=a e^{\lambda x}+b, \quad \psi(x)=c e^{-\lambda x}+d, \quad x \in I,
\end{array}
$$

with some $\lambda \in \mathbb{R} \backslash\{0\}$.

In that case $A_{p}$ is the unique $\left(A_{q}^{[\varphi]}, A_{r}^{[\psi]}\right)$-invariant mean. Moreover, the iterates of $\left(A_{q}^{[\varphi]}, A_{r}^{[\psi]}\right)$ approach $A_{p}$.

Proof. Assume that $\varphi, \psi$ satisfy equation (12) and put $f:=\varphi^{\prime} \circ \varphi^{-1}, g:=$ $\psi^{\prime} \circ \varphi^{-1}$. First assume additionally that $\varphi^{\prime}(x) \neq 0$ and $\psi^{\prime}(x) \neq 0$ for all $x \in I$. Without loss of generality we may assume that $\varphi^{\prime}$ and $\psi^{\prime}$ are positive. By Lemmas 2 and 3 there is a $c_{0} \in(0, \infty)$ such that

$$
f(u)^{q} g(u)^{1-r}=c_{0}, \quad u \in \varphi(I),
$$

and, moreover, $f$ satisfies (16). According to Lemma 4 either

$1^{\circ} f(u)=a, u \in \varphi(I)$, for some $a \in(0, \infty)$,

or

$$
\begin{aligned}
2^{\circ} f(u) & =\lambda(u-b), u \in \varphi(I), \text { with some } \lambda \in \mathbb{R} \backslash\{0\}, b \in \mathbb{R}, \text { and } \\
q+r & =1 .
\end{aligned}
$$

First consider case $1^{\circ}$. Then, by $(25)$, there is a $c \in(0, \infty)$ such that

$$
g(u)=c, \quad u \in \varphi(I) .
$$

Thus

$$
\varphi^{\prime}(x)=a, \quad \psi^{\prime}(x)=c, \quad x \in I,
$$

which completes the proof in case $1^{\circ}$. Now assume $2^{\circ}$. Then, by Lemma 1 , we get $p=1 / 2$. Moreover, from the definition of $f$, we obtain

whence

$$
\varphi^{\prime}(x)=\lambda(\varphi(x)-b), \quad x \in I,
$$

$$
\varphi(x)=a e^{\lambda x}+b, \quad x \in I,
$$


with an $a \in \mathbb{R} \backslash\{0\}$. Now, by (25),

$$
g(u)=-c \lambda \frac{a}{u-b}, \quad u \in \varphi(I),
$$

with a $c \in \mathbb{R} \backslash\{0\}$, which gives

$$
\psi^{\prime}(x)=g(\varphi(x))=-c \lambda e^{-\lambda x}, \quad x \in I,
$$

that is,

$$
\psi(x)=c e^{-\lambda x}+d, \quad x \in I,
$$

with a $d \in \mathbb{R}$.

For the proof in the general case denote by $Z_{\varphi}$ and $Z_{\psi}$ the sets of zeros of $\varphi^{\prime}$ and $\psi^{\prime}$, respectively. It is enough to show that these sets are empty. Since they are closed sets with empty interiors, $I \backslash\left(Z_{\varphi} \cup Z_{\psi}\right)$ is a nonempty open subset of $I$. Let $I_{0}$ be any of its components and suppose that $I_{0} \neq I$. Then at least one end of $I_{0}$, say $x_{0}$, belongs to $I$. Clearly, $x_{0} \in Z_{\varphi} \cup Z_{\psi}$, that is, either $\varphi^{\prime}\left(x_{0}\right)=0$, or $\psi^{\prime}\left(x_{0}\right)=0$. On the other hand, applying the just proved case of the theorem to $\left.\varphi\right|_{I_{0}}$ and $\left.\psi\right|_{I_{0}}$, and the continuity of $\varphi^{\prime}$ and $\psi^{\prime}$ at $x_{0}$, we infer that $\varphi^{\prime}\left(x_{0}\right) \neq 0$ and $\psi^{\prime}\left(x_{0}\right) \neq 0$. This contradiction shows that $I_{0}=I$ and, consequently, $Z_{\varphi}=Z_{\psi}=\emptyset$.

The converse implication can be easily verified. The last paragraph of Theorem 1 is an immediate consequence of Theorem 1 in [6].

Theorem 1 implies the following result concerning equation (1) for general $\alpha$.

Theorem 2. Let $\alpha, \beta, \gamma \in C M(I)$. Assume that $\beta \circ \alpha^{-1}, \gamma \circ \alpha^{-1} \in$ $C^{2} M(I)$. The functions $\alpha, \beta, \gamma$ and numbers $p, q, r \in(0,1)$ satisfy (1) if, and only if, the following two conditions are fulfilled:

(i)

$$
p=\frac{r}{1-q+r},
$$

(ii) there exist $a, c \in \mathbb{R} \backslash\{0\}$ and $b, d \in \mathbb{R}$ such that

$$
\begin{aligned}
& \beta(x)=a \alpha(x)+b, \quad \gamma(x)=c \alpha(x)+d, \quad x \in I, \\
& \text { or } p=1 / 2, q+r=1 \text { and } \\
& \beta(x)=a e^{\lambda \alpha(x)}+b, \quad \gamma(x)=c e^{-\lambda \alpha(x)}+d, \quad x \in I,
\end{aligned}
$$

with some $\lambda \in \mathbb{R} \backslash\{0\}$.

In that case $A_{p}^{[\alpha]}$ is the unique $\left(A_{q}^{[\beta]}, A_{r}^{[\gamma]}\right)$-invariant mean. Moreover, the iterates of $\left(A_{q}^{[\beta]}, A_{r}^{[\gamma]}\right)$ approach $A_{p}^{[\alpha]}$.

Proof. It is enough to observe that $\alpha, \beta, \gamma$ satisfy (1) if, and only if, $\varphi:=\beta \circ \alpha^{-1}$ and $\psi:=\gamma \circ \alpha^{-1}$ satisfy (12) and next use Theorem 1. 


\section{An application}

REMARK. It can be seen from Theorem 1 (or by a direct substitution) that for $q \in(0,1)$ and $\varphi, \psi: I \rightarrow \mathbb{R}$ given by

$$
\varphi(x)=e^{\lambda x}, \quad \psi(x)=e^{-\lambda x}
$$

with arbitrary $\lambda \in \mathbb{R} \backslash\{0\}$ we have

$$
A_{q}^{[\varphi]}(x, y)+A_{1-q}^{[\psi]}(x, y)=x+y, \quad x, y \in I,
$$

that is, the arithmetic mean is the (unique) $\left(A_{q}^{[\varphi]}, A_{1-q}^{[\psi]}\right)$-invariant mean. Moreover, we have

$$
\lim _{n \rightarrow \infty}\left(A_{q}^{[\varphi]}, A_{1-q}^{[\psi]}\right)^{n}(x, y)=\left(\frac{x+y}{2}, \frac{x+y}{2}\right), \quad x, y \in I .
$$

The above Remark allows us to obtain

Theorem 3. Let $q \in(0,1)$ and let $\varphi, \psi: I \rightarrow \mathbb{R}$ be given by (26). $A$ function $F: I^{2} \rightarrow \mathbb{R}$ is a solution of the equation

$$
F\left(A_{q}^{[\varphi]}(x, y), A_{r}^{[\psi]}(x, y)\right)=F(x, y),
$$

continuous at every point of the diagonal $\left\{(x, y) \in I^{2}: x=y\right\}$, if, and only if, there is a continuous function $f: I \rightarrow \mathbb{R}$ such that

$$
F(x, y)=f\left(\frac{x+y}{2}\right), \quad x, y \in I .
$$

Proof. Assume that $F: I^{2} \rightarrow \mathbb{R}$ satisfies (29) and is continuous on the diagonal. By induction we get

$$
F(x, y)=F\left(\left(A_{q}^{[\varphi]}, A_{r}^{[\psi]}\right)^{n}(x, y)\right), \quad x, y \in I, n \in \mathbb{N} .
$$

Letting $n \rightarrow \infty$ and making use of (28) and of the continuity of $F$ at the diagonal we obtain

$$
F(x, y)=F(A(x, y), A(x, y)), \quad x, y \in I .
$$

Putting

$$
f(u):=F(u, u), \quad u \in I,
$$

we get the desired form of $F$. In view of (27) the converse is clear.

Acknowledgements. The authors are indebted to the referee for his valuable comments and remarks, especially those concerning Section 2.

\section{References}

[1] Z. Daróczy and Gy. Maksa, On a problem of Matkowski, Colloq. Math. 82 (1999), $117-123$.

[2] Z. Daróczy and Zs. Páles, On means that are both quasi-arithmetic and conjugate arithmetic, Acta Math. Hungar. 90 (2001), 271-282. 
[3] Z. Daróczy and Zs. Páles, Gauss-composition of means and the solution of the Matkowski-Sutô problem, Publ. Math. Debrecen 61 (2002), 157-218.

[4] M. Kuczma, An Introduction to the Theory of Functional Equations and Inequalities. Cauchy's Equation and Jensen's Inequality, Polish Sci. Publ. and Uniw. Śląski, Warszawa-Kraków-Katowice, 1985.

[5] J. Matkowski, Invariant and complementary quasi-arithmetic means, Aequationes Math. 57 (1999), 87-107.

[6] - Iterations of mean-type mappings and invariant means, Ann. Math. Sil. 13 (1999), 211-226.

[7] O. Sutô, Studies on some functional equations II, Tôhoku Math. J. 6 (1914), 82-101.

Justyna Jarczyk

Faculty of Mathematics

Computer Science and Econometrics

University of Zielona Góra

Szafrana $4 \mathrm{a}$

65-516 Zielona Góra, Poland

E-mail: j.jarczyk@wmie.uz.zgora.pl
Janusz Matkowski Faculty of Mathematics Computer Science and Econometrics University of Zielona Góra Szafrana 4a 65-516 Zielona Góra, Poland E-mail: j.matkowski@wmie.uz.zgora.pl and Institute of Mathematics Silesian University Bankowa 14 40-007 Katowice, Poland

Received 19.5.2005

and in final form 12.11.2005 